\title{
Nutrition, digestive system and digestion specificity in phytophagous bats
}

\author{
NF Zhukova \\ Schmalhausen Institute of Zoology, National Academy of Sciences of Ukraine, \\ B Chme/nitsky Str 15, UA-252601 Kiev, MSP, Ukraine
}

\begin{abstract}
Phytophagous chiropterans occupy a peculiar position among herbivorous mammals. A question has arisen as to how the feeding and digestion specificity is revealed in phytophagous bats, and how the entire order specificity affects these processes.
\end{abstract}

To tackle these issues, the digestive system of the members of the suborder Megachiroptera (Cynopterus sphinx, Rousettus aegyptiacus, Pteropus tonganus) were studied using anatomical and histological methods ; the results obtained were interpreted using certain ecological and physiological data.

The distribution of the representatives of the family Pteropidae, said to be obligate phytophagous, is limited to the tropical and subtropical zones of the Earth. Their food subjects include fruits, nectar, pollen, inflorescence and young leaves of 198 plant genera (Marshall, 1985, Zool J Linn Soc, 83, 4 , 351-369). The distinctive characteristic of their feeding is the mastication of food, within the mouth cavity, when only liquid fraction is swollen.

The digestive system specialisation in phytophagous Pteropidae (especially when an increase of the liquid fraction, rich in carbohydrates and poor in proteins, occurs in the diet), is manifested by : 1) an increase of the stomach-intestinal tract volume at the expense of cardial atrium, fornix and stomach pyloric part volume; certain intestine extension ( 3.5 to 4.6 times exceeding the total body length, as compared to carnivorous bats 1.2 to 3.7 times), including large intestine (19.9 to $23.3 \%$ of the total intestine length, versus 5.2 to $18.0 \%$ in carnivorous species) ; 2) development of the food evacuation out of the stomach slowing down rate mechanism ; 3) development of the optimal digestion and fast assimilation mechanisms (lack of the visible glycocalyx layer, low pancreatic index at increased insular tissue ratio, fructose assimilation predominance over glucose). The origin of the supply of essential amino acids in the obligate phytophagous bat is endogenous protein (during low protein fruit juice forage period).

Because of a significantly conservative digestive system-structure in bats (due to ancestral characteristics retained in different trophic group chiropteran representatives), and the lack of symbiotic digestion in stomach and coecum, the phytophagous chiropterans have developed unique food, consumption, digestion and assimilation mechanisms. Digestive peculiarities of the phytophagous chiropterans, fundamentally different from all other herbivorous mammals, provide high daily food consumption rates (1.4 to 2.5 times exceeding their body mass - Thomas, 1984 , Physiol Zool, 57, 4 457-467), its fast digestion (food passage time 12-44 min - Tedman, Hall, 1985, Austral J Zool, 33, 5, 620-640) and the high level of metabolism (McNab, 1982, Ecology of Bats, NY, L, Plenum Press, chapt 4 , 151-200), all being characteristics of certain importance for the flight locomotion animals. 